\title{
Effect of Amlodipine in Stroke and Myocardial infarction: A Systematic Review and Meta-analysis
}

\author{
Shamanna S. Iyengar · Jagdish Chander Mohan · Saumitra Ray • \\ M. Srinivasa Rao • Mohammed Yunus Khan · Usha Rani H. Patted • \\ Kumar Gaurav
}

Received: May 24, 2021 / Published online: September 4, 2021

(C) The Author(s) 2021

\section{ABSTRACT}

Introduction: Hypertension is a progressive cardiovascular condition arising from complex aetiologies. Progression is strongly associated with functional and structural abnormalities that lead to multi-organ dysfunction. Stroke and myocardial infarction are two of the major complications of hypertension in India. Various anti-hypertensive drugs, such as calcium channel blockers (CCBs), beta-blockers, diuretics, angiotensin-converting enzyme inhibitors and angiotensin receptor blockers, have been the medications of choice for disease management and are known to be effective in reducing the

S. S. Iyengar

Manipal Hospitals, Bangalore, Karnataka, India

J. C. Mohan

Institute of Heart and Vascular Diseases, Jaipur

Golden Hospital, Sector 3, Rohini, Delhi, India

S. Ray

Amri Hospital, Kolkata, West Bengal, India

S. Ray

Woodland Hospital, Kolkata, West Bengal, India

M. S. Rao

Apollo Hospitals, Hyderguda, Hyderabad,

Telangana, India

M. Y. Khan $(\bowtie) \cdot$ U. R. H. Patted · K. Gaurav Medical Affairs, Dr Reddy's Laboratories Ltd., Ameerpet, Hyderabad, Telangana, India e-mail: doctorkhan26@gmail.com complications of hypertension. CCBs, such as amlodipine, are also currently being used and proven to be effective, although their beneficial effects in the management of complications of hypertension like stroke and myocardial infarction (MI) have yet to be proven. Therefore, the aim of this systematic review was to evaluate the effect of amlodipine on stroke and MI in hypertensive patients.

Methods: A systematic search of English electronic databases was performed for studies with sufficient statistical power that were published between 2000 andl 30 August 2020, following the Preferred Reporting Items for Systematic Reviews and Meta-Analyses (PRISMA) criteria. A total of 676 papers were screened, and 13 were found eligible to be included in the meta-analysis. Studies that included patients who suffered from MI or stroke and were under amlodipine treatment were included in the analysis. The odds ratio and the risk ratio of amlodipine compared to active control/placebo were noted from the studies and statistically analyzed.

Results: Amlodipine had a significant effect in reducing stroke and MI in hypertensive patients. Similar to results published in reports, this systematic review proved that the hazard ratio for amlodipine was $<1$ for stroke (0.69-1.04) and MI (0.77-0.98), showing that amlodipine accounted for better prevention of stroke and MI.

Conclusion: In the pooled analysis of data from 12 randomised controlled trials and one double- 
blinded cohort study measuring the effect of CCBs, we found that the CCB amlodipine reduced the risk of stroke and $\mathrm{MI}$ in hypertensive patients. Superior results for amlodipine were found in ten of the 13 studies included in this meta-analysis.

Keywords: Amlodipine; Hypertension; Stroke; Myocardial infarction; Hazard ratio; Risk ratio

\section{Key Summary Points}

Stroke and myocardial infarction (MI) are two significant complications of hypertensive patients in India.

Various anti-hypertensive drugs, such as calcium channel blockers, have been the first choice for managing and effectively reducing the complications of hypertension. Amlodipine, a calcium channel blocker, is also currently being used and has proven to be effective in treating patients with stroke, although its beneficial effects on MI have yet to be demonstrated.

The aim of this systematic review was to evaluate the effect of amlodipine on stroke and MI in hypertensive patients.

Pooled analysis of data from 12 randomised controlled trials and one double-blinded cohort study measuring the effect of amlodipine demonstrated that amlodipine significantly reduced the risk of stroke and MI in hypertensive patients. Superior results for amlodipine were found in ten of the 13 studies included in the systematic review.

\section{INTRODUCTION}

Hypertension is a multi-factorial disorder that results from a complex interplay of environmental and genetic factors. The complexity of blood pressure (BP) control mechanisms has significant implications for an individual's responsiveness to anti-hypertensive drugs, such as like calcium channel blockers (CCBs), beta blockers, angiotensin-converting enzyme (ACE) inhibitors, angiotensin II receptor blockers (ARBs), diuretics, among others.

Uncontrolled hypertension may increase the risk of stroke and myocardial infarction (MI) by fivefold. Stroke and MI are the leading causes of mortality and morbidity in the world, affecting both younger and older individuals [1]. The effective treatment of hypertension and its associated risk factors reduces the risk of hypertensive complications, such as stroke, MI, cardiac failure and renal failure. However, despite current treatment possibilities, hypertension remains a potential risk factor for MI as well as fatal and nonfatal strokes [2]. The authors of a meta-analysis of nine trials concluded that CCBs were associated with a $10 \%$ lower risk of stroke when compared with treatment with different anti-hypertensive drugs [3]. The conclusion arrived at in another metaanalysis was that CCBs, when compared with diuretics and beta-blockers, provided a $13.5 \%$ reduction in the risk of stroke [4].

According to the European Society of Cardiology/European Society of Hypertension (ESCESH) 2018 guideline for arterial hypertension, ACE inhibitors, ARBs, diuretics and CCBs as monotherapy or in combination with each other are used extensively and are listed as the first-line agents for treating hypertension and its complications like stroke and MI [5].

Calcium ions are the culprit that lead to damage of the tissues in the heart and other organs, causing stroke and MI. Massive calcium influx into hypoxic cells is a final common pathway, leading to cell death, and this influx needs to be effectively blocked and managed [6]. Calcium antagonists facilitate this process by decreasing the influx of calcium ions through voltage-sensitive calcium channels [7].

A meta-analysis of 17 randomised controlled trials showed that CCBs reduced the relative risk of stroke by $39 \%$ as compared to placebo [8]. Another meta-analysis that included 6752 patients with a previous history of cerebrovascular disease (stroke or transient ischaemic attack) showed that anti-hypertensive therapy 
resulted in a $28 \%$ reduction in risk for stroke recurrence [9]. Following ischaemic stroke, CCB treatment has been associated with a reduction in mortality (odds ratio [OR] 0.38, 95\% confidence interval [CI] $0.17-0.88$ vs. no CCB treatment) and improvements in the stroke impact scale.

Amlodipine has a strong affinity for cell membranes, modulating calcium influx by inhibiting selected membrane calcium channels. In the landmark ASCOT-BPLA study, amlodipine was associated with a lower risk of stroke than atenolol (hazard ratio [HR] 0.78, 95\% CI 0.67-0.90). These authors also noted that amlodipine was more effective in reducing $\mathrm{BP}$ variability [10]. An analysis of six active controlled trials involving an amlodipine treatment group showed that amlodipine provided more protection against stroke than other antihypertensive agents (OR 0.81, 95\% CI $0.75-0.87 ; \quad P<0.0001)[11,12]$. Similarly, a study performed by Skolnick et al. reported that CCBs have a significant effect on the reduction of MI and its complications [13]. A RCT conducted by Dahlof et al. studied the efficacy of an amlodipine-based regimen compared with an atenolol-based regimen, with the authors ultimately concluding that the patients on the amlodipine-based regimen showed reduction in incidence of fatal stroke (HR 0.90) [14].

The findings of previous studies have proved that CCBs are efficacious medications in terms of hypertension control, as well as the risks of hypertension, such as stroke and MI $[6,7,15]$. Given the high prevalence of hypertension in India, an analysis of current data is essential to obtain a thorough understanding of the properties of CCBs to reduce the risk for stroke and MI. Such information could aid healthcare practitioners in selecting a better treatment option for their hypertensive patients. Therefore, we performed this systematic review and meta-analysis with the aim to evaluate the effect of amlodipine on complications of hypertension, such as stroke and MI.

\section{METHODS}

\section{Search Strategy}

Comprehensive systematic searches of online electronic databases, including PubMed, Cochrane database and Google Scholar (Advanced Google search), were performed to identify studies in which amlodipine was used for the management of stroke and MI. We searched the literature using the following keywords: "Stroke" OR "Cerebro Vascular Accident" OR "CVA" OR "Cerebro Vascular Accident (CVA)" OR "Ischaemic Stroke" OR "Myocardial Infarction" OR "MI" OR "Myocardial Infarction (MI)" OR "Coronary Infarction"; "Amlodipine" OR "FRC-8653)" OR "Calcium Channel Blockers" OR "CCBs"; "Randomised" OR "Randomized" OR "Prospective" OR "Controlled" OR "Uncontrolled" "Blinded" OR "Open Label" OR "Retrospective" OR "Cross-sectional" studies. A total of 676 papers were screened, of which 13 were found to be eligible for inclusion in the study. The studies included participants who suffered from MI or stroke and were receiving amlodipine treatment. The OR and the risk ratio (RR) of amlodipine in comparison to active control/placebo were noted from the studies and statistically analysed.

\section{Study Selection and Data Abstraction}

The search of databases was restricted to English publications. No limitation was set for age of participants in the searched articles. The references of identified papers were screened for any additional article which was not identified in the original search. One reviewer checked all the titles and abstracts and another reviewer checked the article. Both investigators independently reviewed the full text of potential studies for inclusion in the systematic review and no inconsistency was discovered. Hand searching was not performed, and authors of the included articles were not contacted for further information on the studies. 


\section{Quality Assessment}

Eligible studies met the following inclusion criteria: (1) in the case of overlapping studies, then only the study with larger/largest sample size was selected; (2) the numbers of hypertension and stroke and MI cases were provided; (3) data on CCBs and/or placebo were available; (4) the research design was RCTs and parallel arm, double blinded studies, and compared the efficacy of different treatments. Studies were excluded if: they did not provide the ORs, the study population considered was different for different drugs, no specific age criteria were established and study populations were not randomised.

\section{Data Management}

We screened titles, abstracts and full texts according to the inclusion criteria and data were extracted.

\section{Statistical Analysis}

Initially after the screening process had been completed, data extracted from the articles, including the ORs, HRs and RRs, with corresponding 95\% CIs, were entered into an Excel spreadsheet (Microsoft Corp., Redmond, WA, USA). STATA software version 16.0 (StataCorp, College Station, TX, USA) was used for further analysis of the data. We applied logs and converted the values accordingly to the collected ORs, HRs, RRs and 95\% CIs. A standard error variable was estimated based on the log-transformed values of ratios and their corresponding 95\% CI values. Multiple forest plots were generated for stroke and MI using the data collected from the studies based on the ORs, HRs, RRs and respective 95\% CI. Estimated log values and standard error values were also presented obtained along with the weight (\%) given to the studies in the forest plots.

\section{Compliance with Ethics Guidelines}

This article is based on previously conducted studies and does not contain any new studies with human participants or animals performed by any authors.

\section{RESULTS}

\section{Characteristics of the Articles}

Our search identified 676 citations up to 30 August 2020. After a title review and removal of duplicate studies, 584 articles were excluded by screening and 92 full-text articles remained. Some articles did not meet the inclusion criteria; on this basis, 66 full-text papers that had insufficient data for extraction were excluded. After screening for all the probable factors, 13 papers were assessed to be eligible for inclusion in the systematic review. Details on the selection process are shown in Fig. 1

\section{Search Results}

Table 1 provides a summary of the 13 articles included in the systemic review and their methodology. The results of each study are given in the last column toether with the acceptable HRs and ORs. All 13 articles focused on amlodipine and stroke, whereas five articles focused on both MI and stroke with intake of amlodipine. Forest plots were generated to assess the effect of amlodipine treatment on risk reduction of stroke and MI. Head-to-head comparison of available ratios (ORs, HRs, RRs) in the studies selected was performed individually for stroke and MI. Figure 2 provides the ORs for stroke comparing amlodipine and placebo. Figure 3a summarises the HRs of studies on stroke which compared treatment with amlodipine with treatment with other drugs used to control hypertension; Fig. 3b summarises the HRs of studies on stroke in which amlodipine treatment was compared with placebo. Figure 4a summarises the HRs of studies on MI in which amlodipine treatment was compared with treatment with other drugs used 


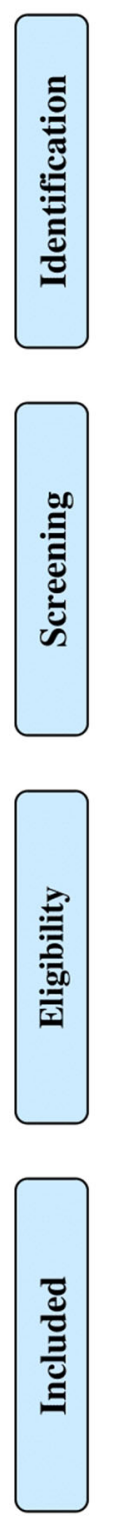

$$
\begin{gathered}
\text { Records identified through database } \\
\text { searching } \\
(\mathrm{n}=655)
\end{gathered}
$$

Additional records identified through other sources $(\mathrm{n}=21)$

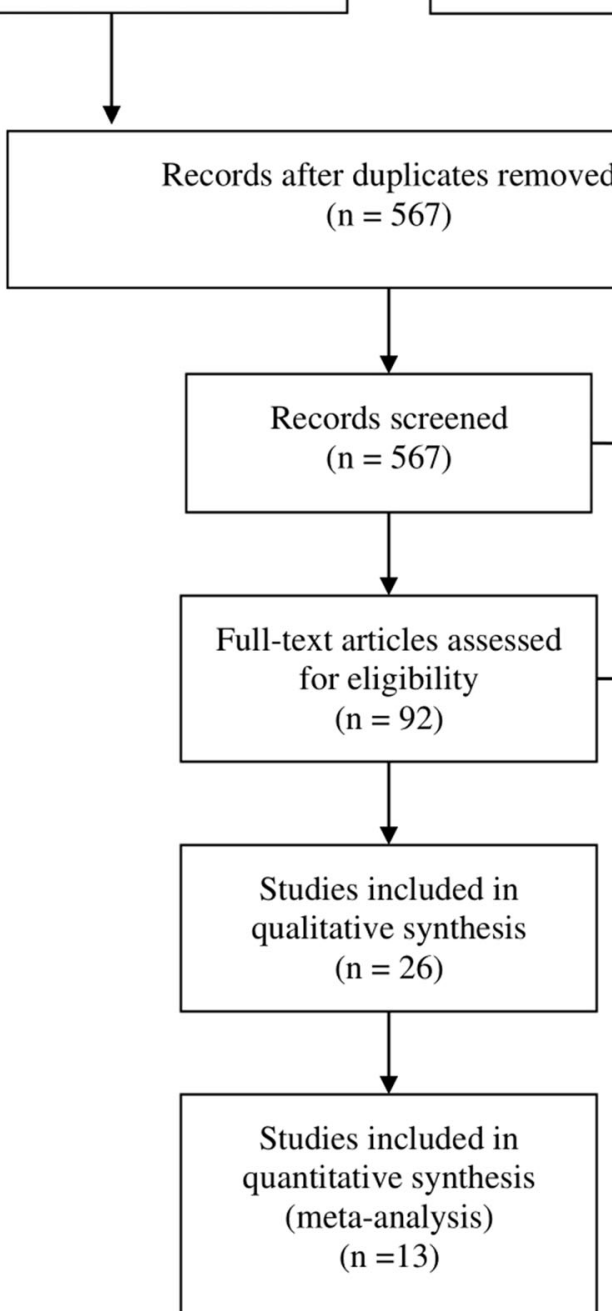

Fig. 1 Flow chart of study selection according to Preferred Reporting Items for Systematic Reviews and Meta-Analyses (PRISMA) guidelines

to control hypertension; Fig. 4b summarises the HRs of studies on MI in which amlodipine treatment was compared with placebo. Figure 5 a summarises the RRs of studies on stroke in which amlodipine treatment was compared with treatment with other drugs used to control hypertension; Fig. $5 \mathrm{~b}$ summarises the RRs of studies on stroke in which amlodipine treatment was compared with placebo. Figure 6 summarises the RR of studies on MI in which amlodipine treatment was compared with placebo.

\section{Publication Bias}

No evidence of publication bias has been found in the current systematic review. 


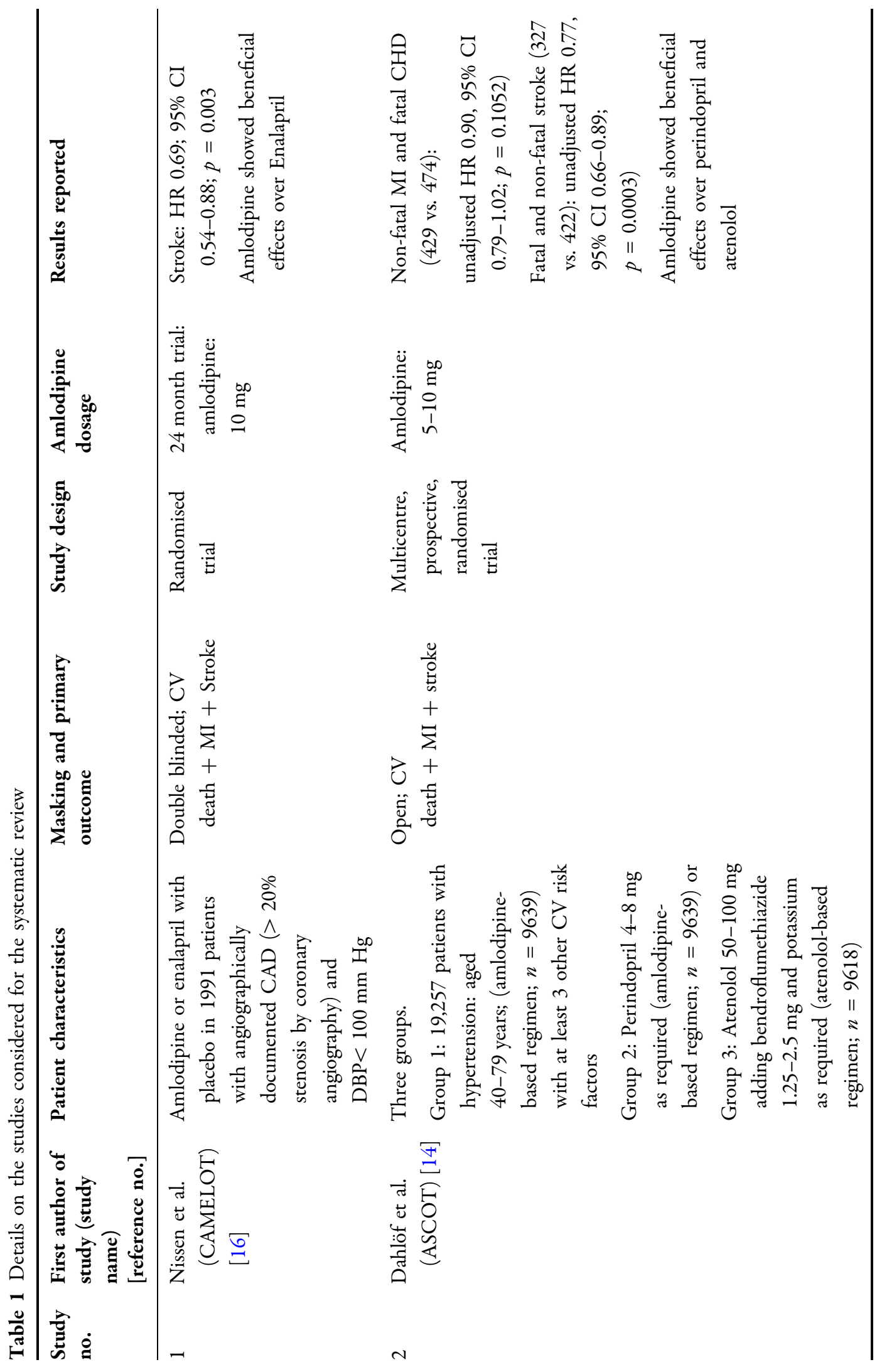




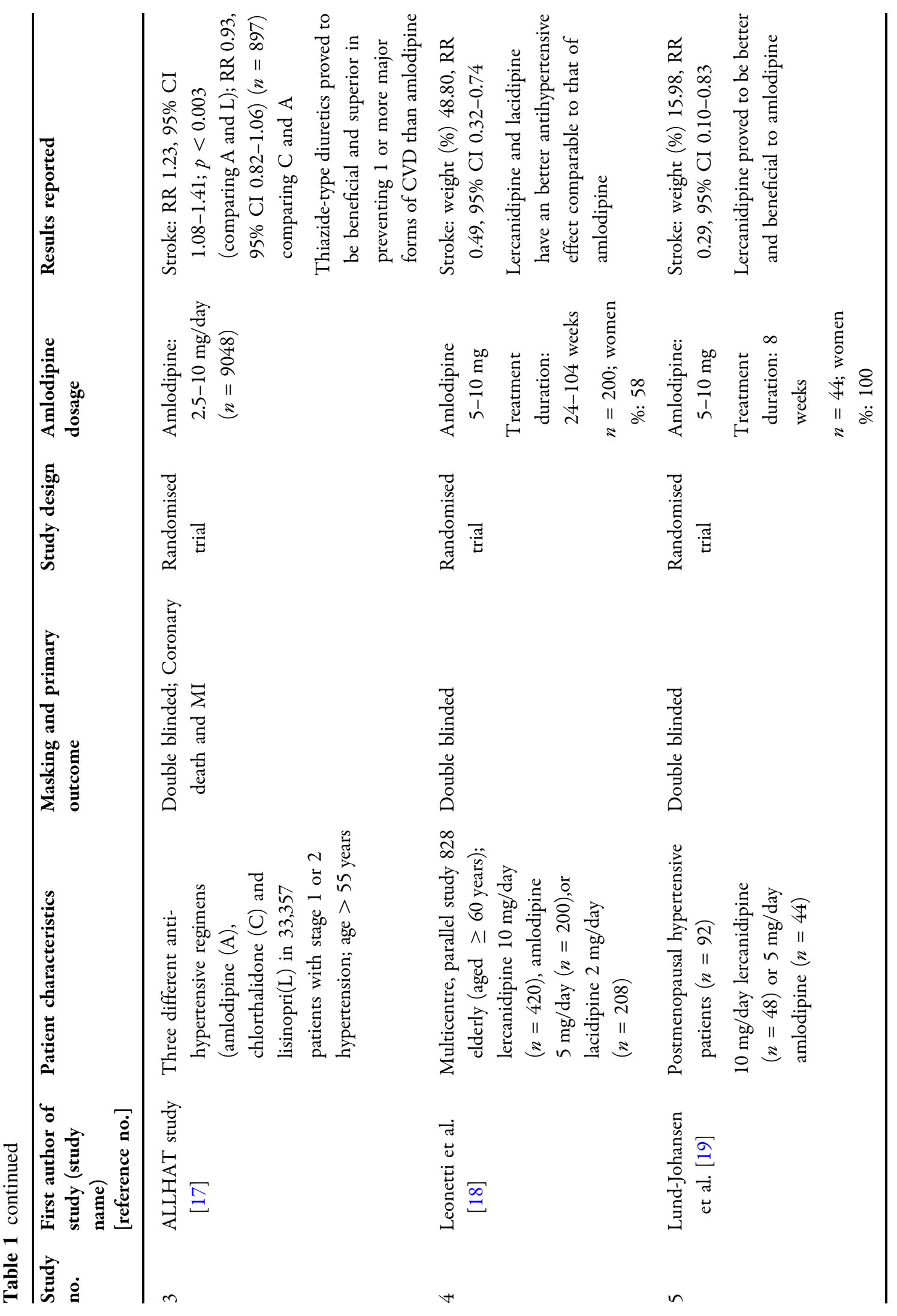




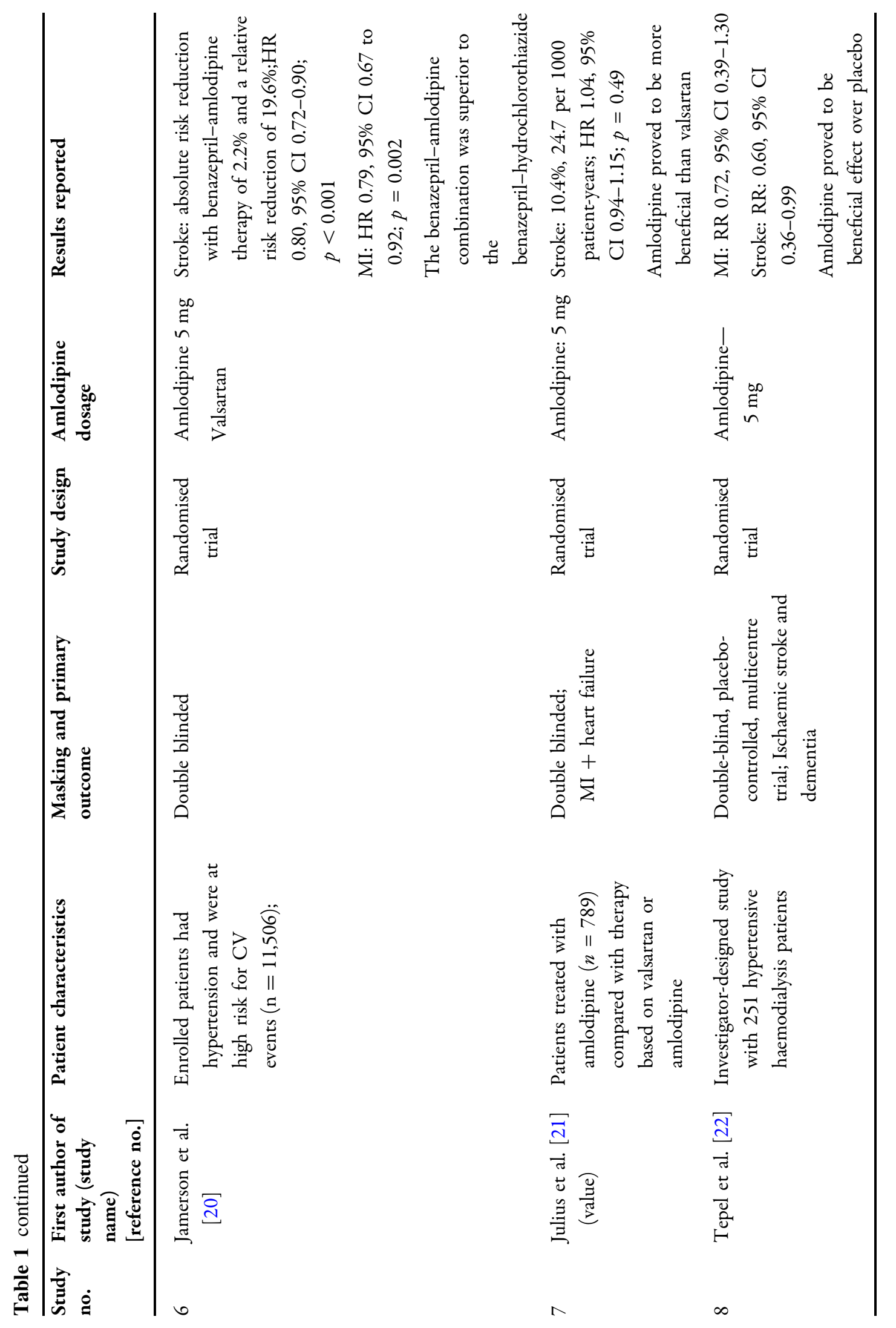




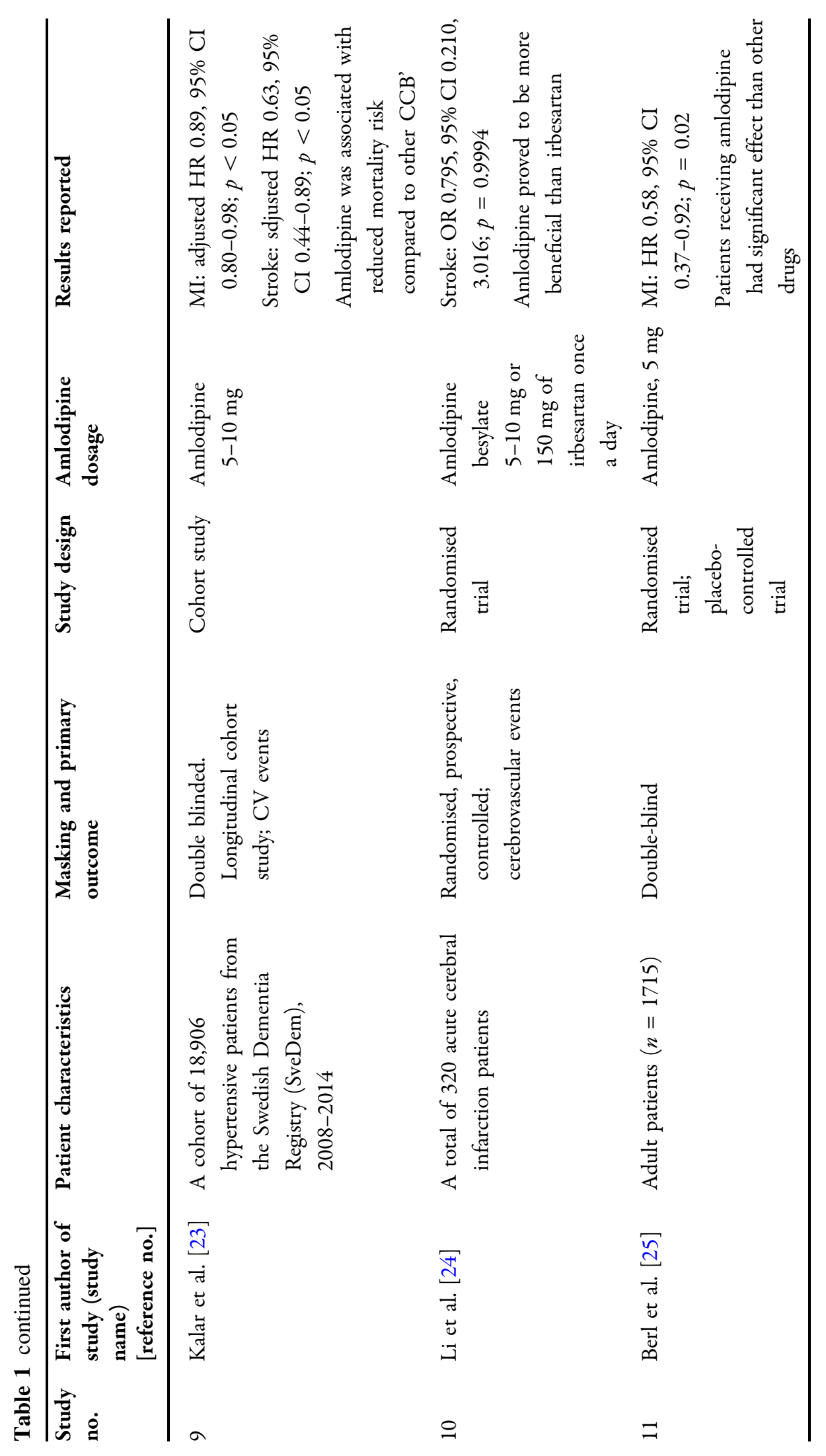




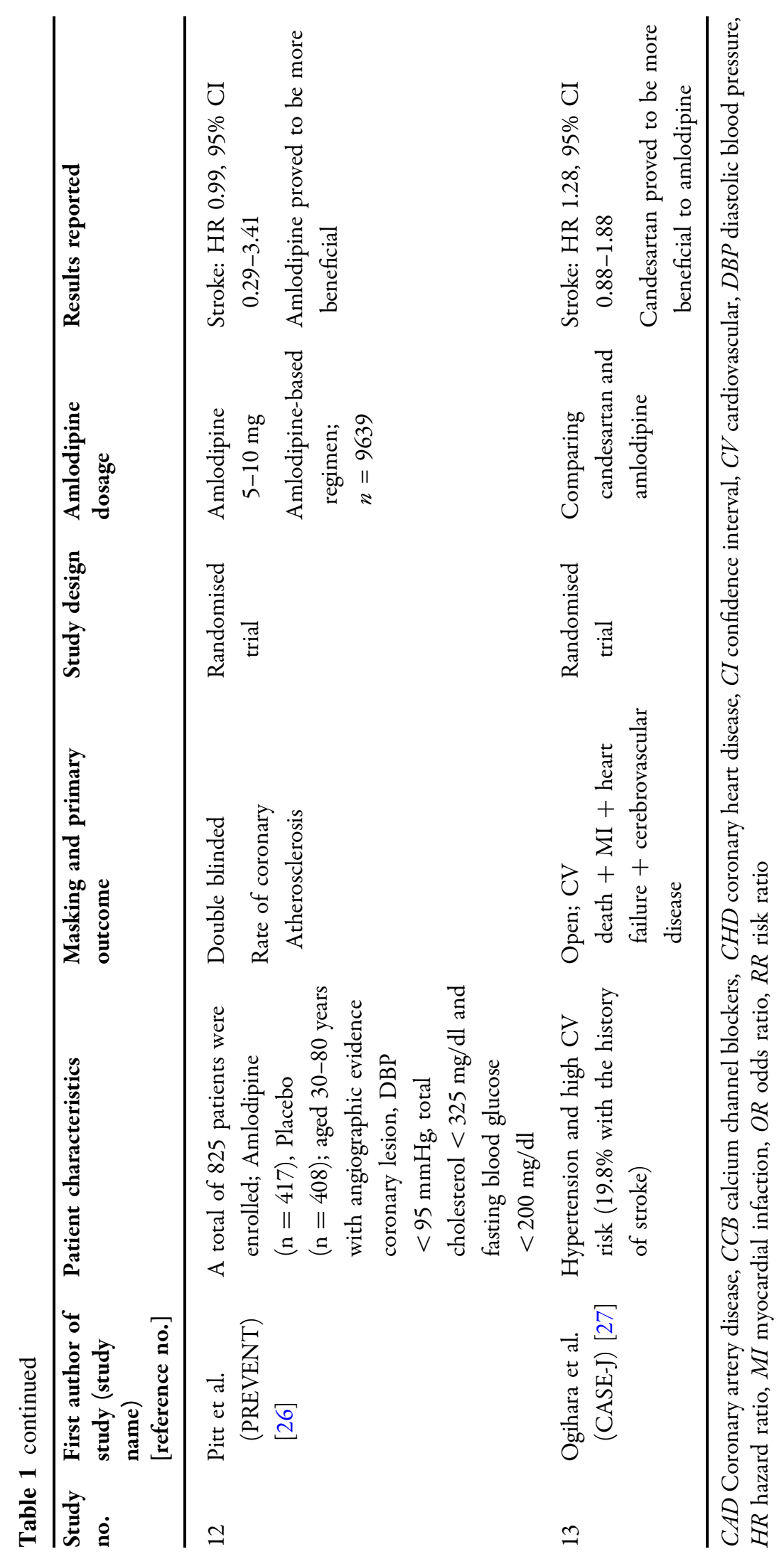




\begin{tabular}{|c|c|c|c|c|c|c|c|c|}
\hline Study or Subgroup & log[Odds Ratio] & SE & Weight & $\begin{array}{c}\text { Odds Ratio } \\
\text { IV, Random, } 95 \% \mathrm{Cl}\end{array}$ & & $\begin{array}{l}\text { Odds Ra } \\
\text { IV, Ranc }\end{array}$ & $\begin{array}{l}\text { o of Stroke } \\
\mathrm{m}, 95 \% \mathrm{Cl}\end{array}$ & \\
\hline Li Y et al. [24] & -0.2294 & 0.6792 & $100.0 \%$ & $0.80[0.21,3.01]$ & & & - & \\
\hline Total $(95 \% \mathrm{Cl})$ & & & $100.0 \%$ & $0.80[0.21,3.01]$ & & & & \\
\hline Heterogeneity: Not applicable & & & & & +1 & & + & 1 \\
\hline Test for overall effect: $Z=0.34(P=0.74)$ & & & & & 0.05 & $\begin{array}{l}0.2 \\
\text { Favours Amlodipine }\end{array}$ & $\begin{array}{lc}1 & 5 \\
\text { Favours Placebo }\end{array}$ & 20 \\
\hline
\end{tabular}

Fig. 2 Forrest plot of the odds ratio and 95\% confidence interval (CI) of stroke (amlodipine vs. placebo). SE Standard error

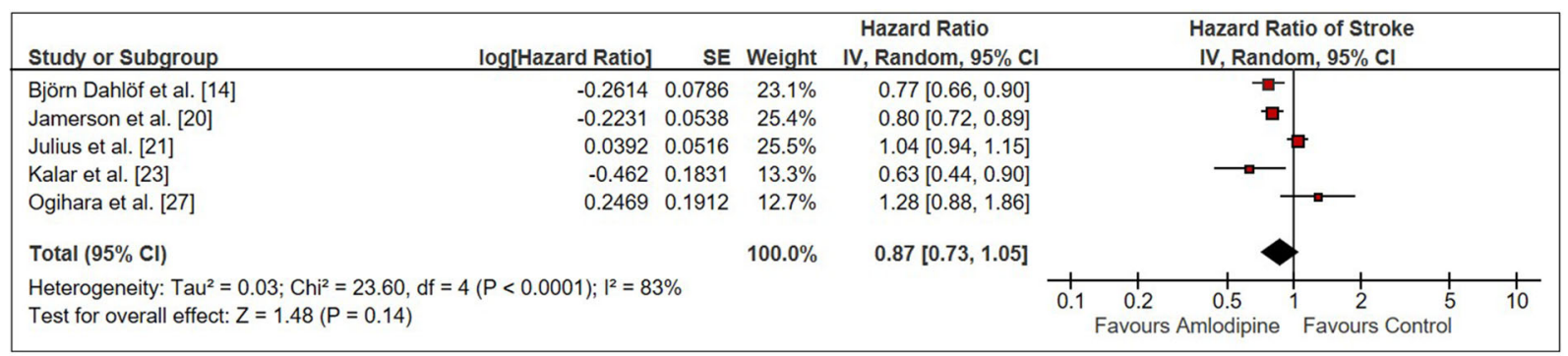

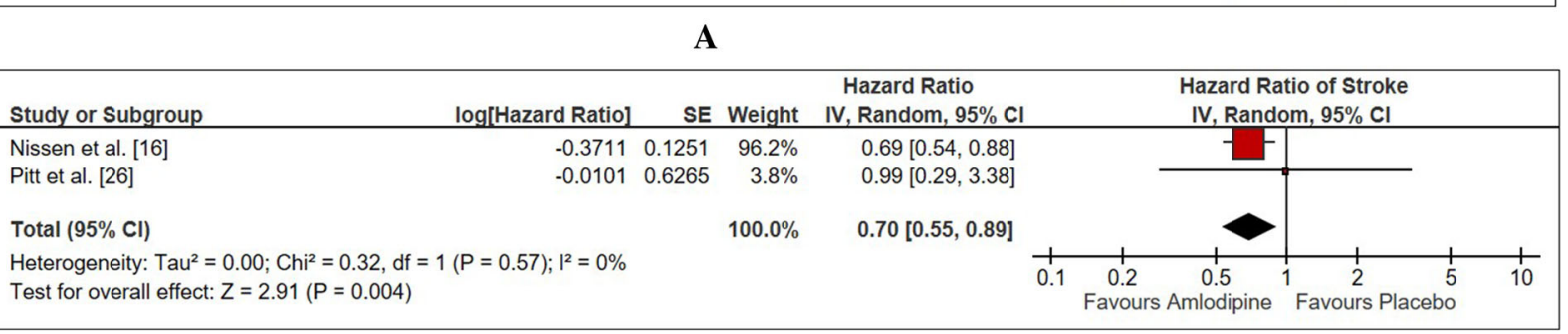

B

Fig. 3 a Forest plot of the hazard ratio (HR) of stroke (amlodipine vs. other drugs), b Forest plot of HR of stroke (amlodipine vs. placebo)

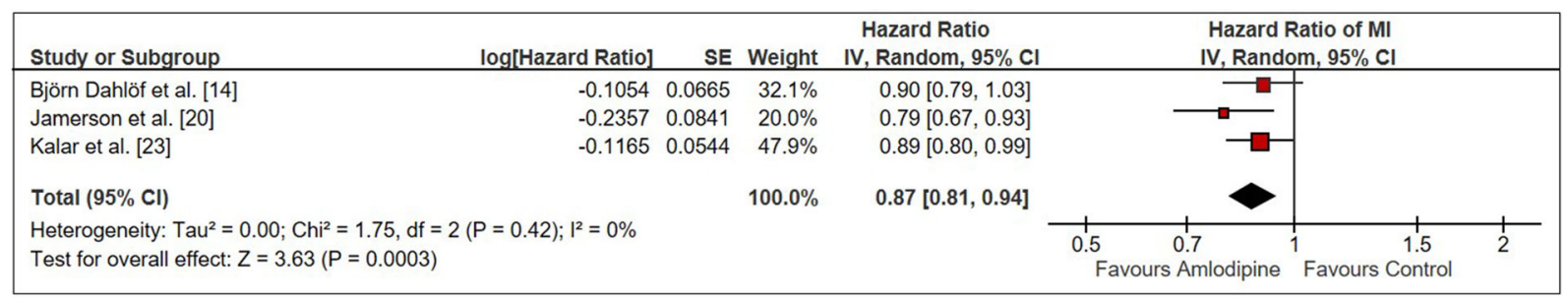

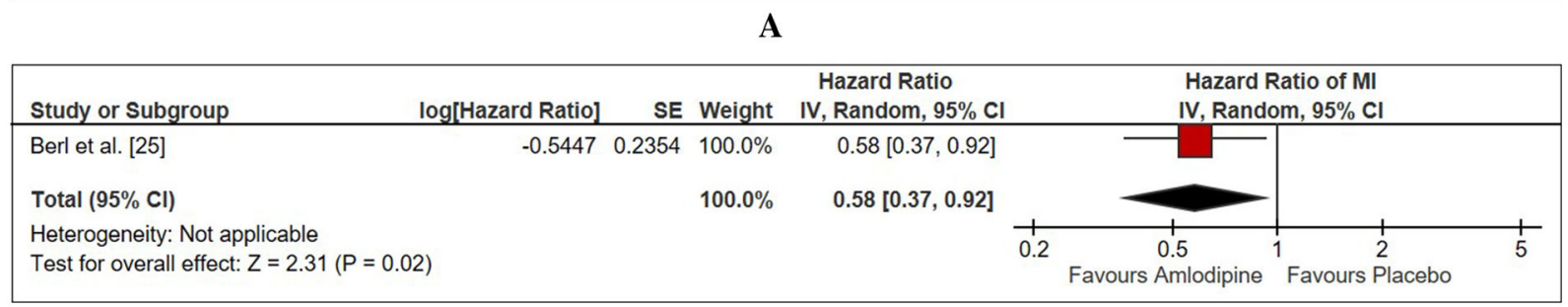

B

Fig. 4 a Forest plot of hazard ratio (HR) of myocardial infarction (MI) (amlodipine vs. other drugs), b Forest plot of HR of MI (amlodipine vs. placebo) 


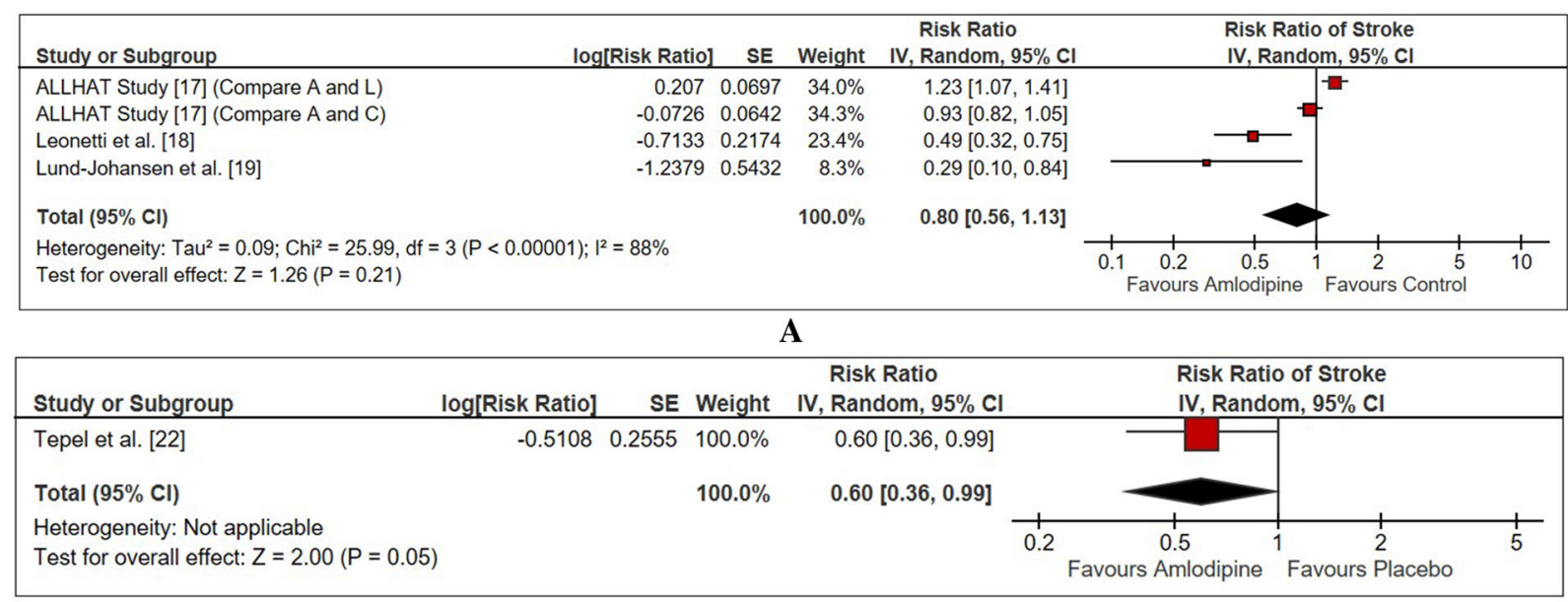

B

Fig. 5 a Forest plot of risk ratio (RR) of stroke (amlodipine vs. other drugs), b Forest plot of RR of stroke (amlodipine vs. placebo)

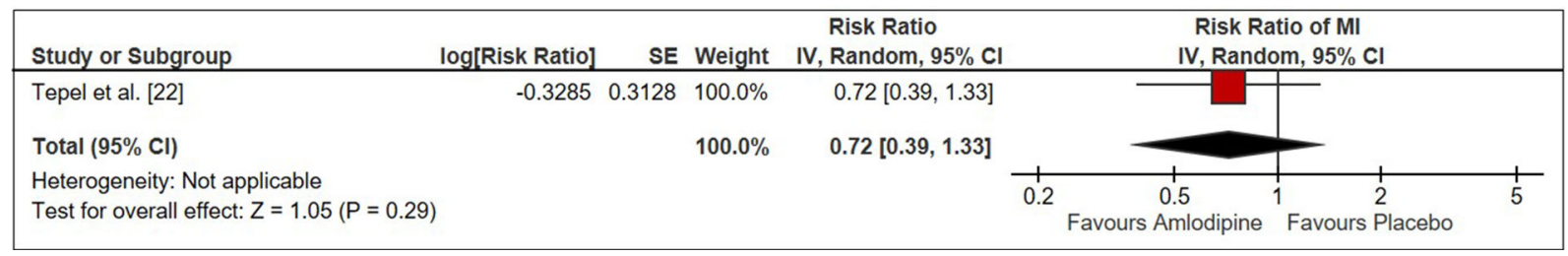

Fig. 6 Forest plot of risk ratio of MI (amlodipine vs placebo)

\section{Meta-Regression Analysis}

Only stroke and MI were taken as drug-modifying aspects or diseases with amlodipine. Multiple factors or diseases were not considered, and meta-regression analysis was not performed.

\section{DISCUSSION}

To the authors' knowledge, this is one of the first study to report the effect of amlodipine on stroke and MI in hypertensive patients. This systematic review and meta-analysis evaluated the impact of amlodipine on stroke and MI in hypertensive patients. CCBs are the drugs of choice for treating hypertension and its complications. Uncontrolled hypertension leads to complications such as stroke and MI, which can be controlled and treated by the appropriate use of anti-hypertensive drugs $[28,29]$.
In this systematic review and meta-analysis, we considered the ORs, HRs, and RRs and constructed forest plots. The results of the selected studies revealed that the risk of stroke and MI events were reduced with the use of CCBs, such as amlodipine. The results presented in the tables of these studies provide new evidence on CCBs that shows that Amlodipine can be beneficial and reduce the incidences of stroke and MI in hypertensive patients.

In the CAMELOT study, a decrease in BP of 3-5 $\mathrm{mmHg}$ in patients receiving amlodipine treatment was accompanied by a $31 \%$ relative reduction in morbidity, although initial BP appeared to be "normal" [18]. The HR and 95\% CI for this study were 0.69 and $0.54-0.88$, respectively ( $p=0.003)$.

In the Valsartan Antihypertensive Long-term Use Evaluation (VALUE) trial, hypertensive patients, of whom 46 and $20 \%$ had a history of cornary heart disease or stroke, respectively, the patients were randomised to an ARB or a CCB 
treatment group. The median follow-up was 4.9 years. The patients who were treated with the ARB valsartan had $2.2 \mathrm{mmHg}$ higher systolic BP than those treated with amlodipine; in addition, the ARB-treated group had a significantly higher rate of stroke and MI by 91 and $15 \%$ compared to the amlodipine group [24].

The overall HR favored amlodipine for the reduction of stroke and $\mathrm{MI}$ in this study.

Cilnidipine is a novel CCB drug which was recently introduced into clinical use. However, clinical data on long-term outcomes trials on stroke and MI with cilnidipine are still lacking. Cilnidipine is also a long-acting CCB, like amlodipine, but it has a shorter half-life (2.1-2.5 vs. $35-50 \mathrm{~h})$ [30]. Amlodipine has an excellent pharmacokinetic and pharmacodynamic profile and provides with good BP control for $24 \mathrm{~h}$ longer than cilnidipine. The varied effects of its anti-anginal and anti-atherosclerotic properties have made it a classical drug of choice compared with the newer CCBs like cilnidipine [30]. Amlodipine is relatively cheaper than cilnidipine and minimises BP variability, thereby reducing the risk of cardiovascular and cerebrovascular events, whereas data from human trials with cilnidipine are still lacking [31]. Due to limited data available on the effect of cilnidipine on stroke and MI, a comparative analysis with amlodipine was not performed in the present systematic review.

Multiple published research studies have proven the advantages of amlodipine over other drugs. As evidenced by the ALLHAT [14] and ASCOT [20] studies, an amlodipine-based antihypertensive regimen might confer more outcome benefits than other drug regimens, possibly by lowering central systolic BP by $4.3 \mathrm{~mm}$ of $\mathrm{Hg}$ [32] or ambulatory BP over $24 \mathrm{~h}$. It is also beneficial in reducing nocturnal $\mathrm{BP}$ and prevents intima-media thickening. Another reason for using amlodipine is its effect on systolic BP variability, a recognised risk factor, and marker for cardiovascular diseases.

\section{Limitations of the Study}

This study has a number of limitations. Although this meta-analysis included all long- term outcome trials, small-scale intermediate endpoint studies were excluded, possibly resulting in a deviation from the meta-analysis. The definition of cardiovascular events and stroke differed between the studies. Only studies of the English language were included in this review which might make it open to language bias. Also, the treatment follow-up duration of studies included varied greatly, which could result in bias.

One additional limitation found with this study that deserves mention is the use of different ratios, such as RR, HR and OR. Different studies reported different risks, i.e. uniformity was not maintained. Risk in the present study was minimised through an extensive search of the available literature. Although the statistical tests did not indicate publication bias, there is clearly limited power to detect such bias, given the relatively small number of studies examined. More studies with fewer limitations should be conducted to verify the results of the present study.

\section{CONCLUSION}

Findings of the present meta-analysis lead to the conclusion that amlodipine has beneficial effects in reducing the incidence of stroke and MI in hypertensive patients. Its high tolerability and minimal side effects compared to other classes of anti-hypertensive drugs have made it an agent of choice in both single and combination drug treatment to reduce the burden of stroke and MI across the globe. The results from this systematic review and meta-analysis may help physicians determine the best treatment options for their hypertensive patients for stroke and MI prevention. However, more studies are required to corroborate the findings of our study.

\section{ACKNOWLEDGEMENTS}

Compliance with Ethics Guidelines. This article is based on previously conducted studies and does not contain any new studies with 
human participants or animals performed by any authors.

\section{Medical Writing, Editorial and Other} Assistance. The authors thank Dr. Shri L (Medical Writer) from QREC Clinical Research LLP, India for support in the preparation of the manuscript.

Funding. The study was funded by Dr Reddy's Laboratories. The sponsor is also funding Journal's Rapid Service fee.

Disclosures. This study represents original work. It was completed recently and has not been published elsewhere. All the authors of this paper have contributed equally to the work and preparation of the manuscript. All authors, Shamanna S Iyengar, Jagdish C Mohan, Saumitra Ray and Srinivasa Rao $\mathrm{M}$ are on the advisory board members for Dr Reddy's Laboratories Ltd. Yunus Khan, Kumar Gaurav and Usha Rani Patted declare that they work in the Medical Affairs Department in Dr Reddy's Laboratories Ltd, Hyderabad, India.

Authorship. All named authors meet the International Committee of Medical Journal Editors (ICMJE) criteria for authorship for this article, take responsibility for the integrity of the work as a whole, and have given their approval for this version to be published. All the authors of this paper have contributed equally to the work and preparation of the manuscript.

Authorship Contributions. All authors contributed to the study conception and design. Material preparation, data collection and analysis were performed by Mohammed Yunus Khan, Usha Rani H Patted and Kumar Gaurav. Shamanna S Iyengar, Jagdish Chander Mohan, Saumitra Ray and Srinivasa Rao M reviewed and approved the final results of analysis. The first draft of the manuscript was written by Mohammed Yunus Khan, Usha Rani H Patted and Kumar Gaurav and all authors commented on previous versions of the manuscript. All authors read and approved the final manuscript.
Data Availability. Data sharing is not applicable to this article as no datasets were generated or analyzed during the current study.

Open Access. This article is licensed under a Creative Commons Attribution-NonCommercial 4.0 International License, which permits any non-commercial use, sharing, adaptation, distribution and reproduction in any medium or format, as long as you give appropriate credit to the original author(s) and the source, provide a link to the Creative Commons licence, and indicate if changes were made. The images or other third party material in this article are included in the article's Creative Commons licence, unless indicated otherwise in a credit line to the material. If material is not included in the article's Creative Commons licence and your intended use is not permitted by statutory regulation or exceeds the permitted use, you will need to obtain permission directly from the copyright holder. To view a copy of this licence, visit http://creativecommons.org/licenses/by$\mathrm{nc} / 4.0 /$.

\section{REFERENCES}

1. Sacco RL, Kasner SE, Broderick JP, et al. An updated definition of stroke for the 21st century: a statement for healthcare professionals from the American Heart Association/American Stroke Association. Stroke. 2013;44:2064-89.

2. Bronner LL, Kanter DS, Manson JE. Primary prevention of stroke. N Engl J Med. 1995;23:1392-400.

3. Pahor M, Psaty BM, Alderman MH, et al. Health outcomes associated with calcium antagonists compared with other first-line anti-hypertensive therapies: a meta-analysis of randomized controlled trials. Lancet. 2000;356:1949-54.

4. Staessen JA, Wang JG, Thijs L. Cardiovascular prevention and blood pressure reduction: a quantitative overview updated until March 1st 2003. J Hypertens. 2003;21:1055-76.

5. Williams B, Mancia G, Spiering W, et al. 2018 ESC/ ESH Guidelines for the management of arterial hypertension The Task Force for the management of arterial hypertension of the European Society of Cardiology (ESC) and the European Society of 
Hypertension (ESH). Eur Heart J 2018;39(33): 3021-104.

6. Siesjo BK, Bengtsson F. Calcium fluxes, calcium antagonists and calcium-related pathology in brain ischemia, hypoglycemia and spreading depression: a unifying hypothesis. J Cereb Blood Flow Metab. 1989;9:127-40.

7. Rami A, Krieglstein J. Neuronal protective effects of calcium antagonists in cerebral ischemia. Life Sci. 1994;55:2105-13.

8. Lawes CM, Bennett DA, Feigin VL, Rodgers A. Blood pressure and stroke: an overview of published reviews. Stroke. 2004;35(4):1024-33.

9. Gueyffier F, Boissel JP, Boutitie F et al. Effect of antihypertensive treatment in patients having already suffered from stroke. Gathering the evidence. The INDANA (INdividual Data ANalysis of Antihypertensive intervention trials) Project Collaborators. Stroke. 1997;28(12):2557-62.

10. Wang JG, Li Y, Franklin SS, Safar M. Prevention of stroke and myocardial infarction by amlodipine and angiotensin receptor blockers. A quantitative overview. Hypertension. 2007;50(1):181-8.

11. Schofer J, Spielmann R, Schuchert A, Weber K, Schluter M. Iodine-123 meta-iodobenzylguanidine scintigraphy: a noninvasive method to demonstrate myocardial adrenergic nervous system disintegrity in patients with idiopathic dilated cardiomyopathy. J Am Coll Cardiol. 1988;12:1252-8.

12. Nagahama S, Norimatsu T, Maki T, et al. The effect of combination therapy with an L/N-Type $\mathrm{Ca}(2+)$ channel blocker, cilnidipine, and an angiotensin II receptor blocker on the blood pressure and heart rate in Japanese hypertensive patients: an observational study conducted in Japan. Hypertens Res. 2007;30:815-22.

13. Skolnick AE, Frishman WH. Calcium channel blockers in myocardial infarction. Arch Intern Med. 1989;149(7):1669-77.

14. Dahlöf B, Sever PS. Prevention of cardiovascular events with an anti-hypertensive regimen of Amlodipine adding perindopril as required versus atenolol adding bendroflumethiazide as required, in the Anglo-Scandinavian Cardiac Outcomes TrialBlood Pressure Lowering Arm (ASCOT-BPLA): a multicentre randomized controlled trial. Lancet. 2005;366:895-906.

15. Elliott WJ, Bandari A. The role of calcium antagonists in stroke prevention. J Clin Hypertens (Greenwich). 2005;7(4 Suppl 1):5-8.
16. McDonald MA, Simpson SH, Ezekowitz JA, Gyenes G, Tsuyuki RT. Angiotensin receptor blockers and risk of myocardial infarction: systematic review. BMJ. 2005;331(7521):873.

17. Pavasini R, et al. Complete revascularisation reduces cardiovascular death in patients with ST-segment elevation myocardial infarction and multivessel disease: systematic review and metaanalysis of randomized clinical trials. Eur Heart J. 2020;41(42):4103-10.

18. Nissen SE, Tuzcu EM, Libby P, et al. Effect of antihypertensive agents on cardiovascular events in patients with coronary disease and normal blood pressure: the CAMELOT study: a randomized controlled trial. JAMA. 2004;292(18):2217-25.

19. Strauss $\mathrm{MH}$, Hall AS. Angiotensin receptor blockers do not reduce risk of myocardial infarction, cardiovascular death, or total mortality: further evidence for the ARB-MI paradox. Circulation. 2017;135(22):2088-90.

20. The ALLHAT Officers and Coordinators for the ALLHAT Collaborative Research Group. Major outcomes in high-risk hypertensive patients randomized to angiotensin-converting enzyme inhibitor or calcium channel blocker vs diuretic: the anti-hypertensive and lipid-lowering treatment to prevent heart attack trial (ALLHAT). JAMA. 2002;288(23): 2981-97.

21. Leonetti G, Magnani B, Pessina AC, et al. Tolerability of long-term treatment with lercanidipine versus Amlodipine and lacidipine in elderly hypertensives. Am J Hypertens. 2002;15:932-40.

22. Lund-Johansen P, Stranden E, Helberg S, et al. Quantification of leg oedema in postmenopausal hypertensive patients treated with lercanidipine or amlodipine. J Hypertens. 2003;21:1003-10.

23. Jamerson K, Weber MA, Bakris GL, et al. Benazepril plus amlodipine or hydrochlorothiazide for hypertension in high-risk patients. $\mathrm{N}$ Engl J Med. 2008;359:2417-28.

24. Julius S, Kjeldsen SE, Weber M, et al. Outcomes in hypertensive patients at high cardiovascular risk treated with regimens based on valsartan or amlodipine: the VALUE randomized trial. Lancet. 2004;363:2022-31.

25. Tepel M, Hopfenmueller W, Scholze A, Maier A, Zidek W. Effect of amlodipine on cardiovascular events in hypertensive haemodialysis patients. Nephrol Dial Transplant. 2008;23:3605-12.

26. Kalar I, Xu H, Secnik J, et al. Calcium channel blockers, survival and ischaemic stroke in patients 
with dementia: a Swedish registry study. J Intern Med. 2021;289(4):508-22.

27. Li Y, Zhong Z, Luo S, et al. Efficacy of anti-hypertensive therapy in the acute stage of cerebral infarction-a prospective, randomized control trial. Acta Cardiol Sin. 2018;34(6):502-10.

28. Berl T, Hunsicker LG, Lewis JB, et al. Cardiovascular outcomes in the Irbesartan Diabetic Nephropathy Trial of patients with type 2 diabetes and overt nephropathy. Ann Intern Med. 2003;138(7):542-9.

29. Pitt B, Byington RP, Furberg CD, et al. Effect of amlodipine on the progression of atherosclerosis and the occurrence of clinical events. PREVENT Investigators. Circulation. 2000;102(13):1503-10. https://doi.org/10.1161/01.cir.102.13.1503.
30. Tiwaskar M, Langote A, Kashyap R, Toppo A. Amlodipine in the era of new generation calcium channel blockers. J Assoc Physicians India. 2018;66(3):64-9.

31. Shetty K, Shetty R, Bairy L, et al. A comparative study on clinical and biochemical parameters in amlodipine and cilnidipine treated hypertensive patients. J Clin Diagn Res. 2017;11(5):FC01-5. https://doi.org/10.7860/JCDR/2017/23825.9763.

32. The CAFE Investigators. Differential impact of blood pressure-lowering drugs on central aortic pressure and clinical outcomes. Principal results of the Conduit Artery Function Evaluation (CAFE) study. Circulation. 2006;113:1213-25. 